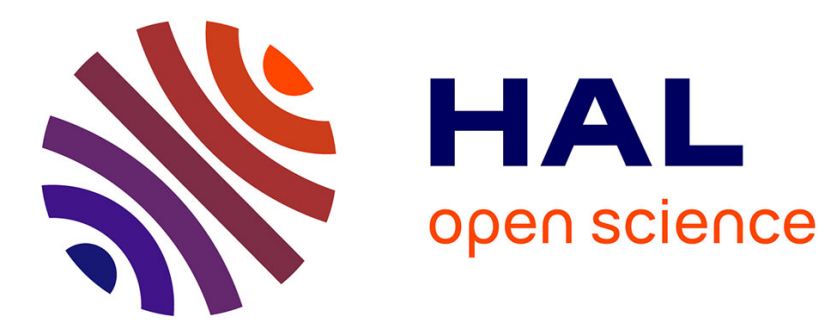

\title{
Identification of a low-frequency elastic behaviour in liquid water
}

Laurence Noirez, Patrick Baroni

\section{To cite this version:}

Laurence Noirez, Patrick Baroni. Identification of a low-frequency elastic behaviour in liquid water . Journal of Physics: Condensed Matter, 2012, 24 (37), pp.372101. 10.1088/0953-8984/24/37/372101 . hal-01295302

\section{HAL Id: hal-01295302 \\ https://hal.science/hal-01295302}

Submitted on 9 Sep 2016

HAL is a multi-disciplinary open access archive for the deposit and dissemination of scientific research documents, whether they are published or not. The documents may come from teaching and research institutions in France or abroad, or from public or private research centers.
L'archive ouverte pluridisciplinaire HAL, est destinée au dépôt et à la diffusion de documents scientifiques de niveau recherche, publiés ou non, émanant des établissements d'enseignement et de recherche français ou étrangers, des laboratoires publics ou privés. 


\title{
Identification of a low frequency elastic behavior in Liquid Water
}

\author{
Laurence Noirez and Patrick Baroni \\ Laboratoire Léon Brillouin (CEA-CNRS) \\ CE-Saclay \\ 91191 Gif-sur-Yvette Cédex \\ France \\ E-mail: laurence.noirez@cea.fr. Tel: 331690863 00, Fax: 33169088251.
}

\begin{abstract}
This article deals with the identification of solid-like properties measured at room temperature at a sub-millimeter length scale in liquid water. At a macroscopic scale, normal liquids (i.e above the melting temperature of the crystal) and in particular water, are typically and empirically defined by the absence of shear elasticity by opposition to solids or plastic fluids that require a stress threshold for flowing. A novel method optimising the transmission of the shear stress to the sample enables a more complete probe of the mechanical response of liquids. It reveals that glass formers and viscous alkanes exhibit actually finite macroscopic shear elasticity away from any phase transition. This protocol is here applied for the first time to liquid water at room temperature revealing, at sub-millimetre scale, a low-frequency solidlike property.
\end{abstract}

Keywords: liquid water, sub-millimetre scale, macroscopic shear elasticity, solid-like correlations, primary elastic regime.

\section{Introduction :}

On the basis of a Maxwell gas model (1867), it has long been suspected that liquids exhibit (shear) elasticity only at sufficiently high solicitation frequencies $(\mathrm{MHz}$ or $\mathrm{GHz})$. Recent experimental improvements show that actually it is possible to measure also shear elasticity at low frequency $(0.01$ to $16 \mathrm{~Hz}$ ) and at sub-millimeter scale. In other words, liquids exhibit long range solid-like correlations at a macroscopic scale away from any phase transition.

We probe here the low frequency dynamic response of liquid water and show for the first time an elastic response at a sub-millimeter thickness. This result has been enabled by applying a weak mechanical stress efficiently transmitted to the liquid by improving the boundary conditions between liquid and the substrate. These conditions preserve the liquid as much as possible close to its equilibrium state. This protocol established since 2005 has enabled to measure a several decades higher mechanical response independent of the frequency in melts of standard polymers[1-2]. So far neglected shear elasticity (i.e. solid-like component) was highlighted far away from the glass transition and at a macroscopic scale. Later, the observation of a low frequency shear elasticity above the melting point in glass formers as Glycerol [3] or o-terphenyl[4], and more recently in a viscous liquid heptadecane [5], invalidates an interpretation in terms of dynamic heterogeneities (clusters) reminiscent of the glass transition[6,7]. The low frequency shear elasticity has the characteristics of a generic condensed matter property (i.e. at thermodynamical equilibrium).

Here, we describe the first experiments carried out on liquid water with this method.

Several decades of physical, chemical, biological, thermodynamical, interfacial studies has certainly made water the most studied liquid [8]. It has been in particular established that the confined geometry (capillaries, at interface, in thin layers, or in interactions with biological systems) leads to a slowing down of dynamic with respect to the "bulk" properties[8-10]. Mechanical measurements reveal scaledependent interactions at molecular scale or at a multiple of that as reported for example in Surface Forces Apparatus experiments[9,10]. These studies are restricted to several molecular thick layers, i.e. typically layers of much less than the micron scale. Several authors report on an increased viscosity at 
$1000-10000 \AA$ length scale away from the surface. These results were interpreted by surface induced effects or interfacial phenomena [9,10] and some by intrinsic properties[12]. The mechanical approaches (SFA, flow measurements) were mainly carried out without considerations of the interactions of the liquid to the substrate. By improving these boundary interactions, the present results on liquid water report on elastic properties at several hundreds microns length scale (Fig.4) and invite to rethink how the liquid state should be considered.

\section{Experimental :}

The dynamic probe is a soft mechanical stress as non-disturbing as possible to keep intact the material properties (linear conditions). The study is focussed on the low frequency response $\left(10^{-1}-10^{2} \mathrm{rad} / \mathrm{s}\right)$, applying therefore low shear strain stresses $(0.1 \%-2 \%)$. This dynamic mechanical analysis belongs to the rare methods giving access to slow relaxation times.

This method consists in applying a small shear stress/strain to the fluid via surface contact. A strong interaction between the liquid and the substrate is obtained by using a zero-porosity alumina substrate[13]. It optimizes the liquid/substrate interaction by promoting the full wetting of the liquid on the surfaces and expulsing the eventual interfacial gas layer between the liquid and the substrate (total wetting conditions[14]).

The sample (distilled water) placed in the gap of two wetting surfaces is submitted to a small shear solicitation of constant shear strain $\gamma_{0}$ (imposed strain mode). Its response is measured via the shear stress transmitted by the sample to the second surface (immobile and coupled to a sensor). Reported data correspond to macroscopic measurements carried at room temperature and $0.125 \mathrm{~mm}$ gap thickness.

The oscillatory motion and the shear stress measurement are provided by a conventional rheometer (ARES2) equipped with alumina plates of $40 \mathrm{~mm}$ diameter. This device has been equipped with a 7digits voltmeter (Keitley - Rate: 300 data/s) that simultaneously measures the voltage of the motor imposing the oscillation (input wave), while another 7-digits voltmeter measures the voltage associated to the sensor (output wave). This setup enables the simultaneous access to the strain/stress signals and to the dynamic profile using the conventional formalism following the relationship: $\sigma(\omega)=$ $\gamma_{0} \cdot \sin (\omega . t+\Delta \phi)$ with $\sigma(\omega)$ the shear stress and $\Delta \phi$ the phase shift between the input and the output waves, or in terms of shear elastic $\left(G^{\prime}\right)$ and viscous $\left(G^{\prime \prime}\right)$ moduli: $\sigma(\omega)=\gamma_{0 .}\left(G^{\prime}(\omega) \cdot \sin (\omega . t)+\right.$ $\left.G^{\prime \prime}(\omega) \cdot \cos (\omega \cdot t)\right)$, with $G$ ' the component in phase with the strain, and $G$ " the out of phase component. A perfect solid-like response displays no phase shift in the output response. Experimentally, solid-like materials exhibit always a short delay due to internal freedom degrees. As long as the phase shift remains lower than $\Delta \phi<\pi / 4$, the elastic component $G$ ' dominates the viscous component $G$ "(Fig.1a). If the output response of the material presents a phase shift larger than $\pi / 4$, then the viscous component $G$ " dominates the elastic component $G^{\prime}$ (Fig.1b). The sample exhibits a liquid-like behaviour. If the phase shift reaches $\pi / 2$ ( $G^{\prime}$ vanishes), the sample is defined as purely viscous.

The sample response can be scanned as a function of the frequency $(\omega)$, typically from $10^{-1} \mathrm{rad} / \mathrm{s}$ up to $10^{2} \mathrm{rad} / \mathrm{s}$ (i.e. probing time scales from $0.01 \mathrm{~s}$ up to $10 \mathrm{~s}$ ) giving the frequency range over the sample responds elastically or in a dissipative way (viscous behaviour).

A solid-like behaviour is independent of the frequency whereas a (Maxwell) liquid-like behaviour displays typically a vanishing response characterised by a $\omega$-scaling of the viscous modulus and a $\omega^{2}$ scaling dependence of the elastic modulus. A purely viscous sample does not display any elastic component.

Finally, it should be stressed that the formalism in terms of $G^{\prime}$ and $G$ " imposes that the resulting stress wave keeps the shape of the imposed strain wave (sinusoidal-like). The study of the signal will show that this condition is actually valid in two extreme situations only, corresponding to the very weak and to the high strain fields respectively. 


\section{Results:}

\subsection{Dynamic relaxation measurements:}

Fig.2a illustrates the input sinusoidal wave and the output shear stress transmitted by a layer of $0.125 \mathrm{~mm}$ of liquid water over four oscillation periods. The liquid water is solicited at $24^{\circ} \mathrm{C}$ at $1 \mathrm{low}$ strain amplitude $\left(\gamma_{0}=3 \%\right)$; i.e. the mechanical perturbation is minimized, using total wetting boundary conditions. Fig.2a shows that both input and output waves are almost superposed indicating a quasiinstantaneous sample response $\left(\Delta \phi<8^{\circ}\right)$, i.e. a solid-like response. This result indicates that when the liquid is firmly anchored on the surfaces, its response to a weak mechanical solicitation is solid-like. A similar in-phase signal is observed over the wide range of frequency tested in Fig.2b. Since the output shear stress is sinusoidal, a description in terms of elastic $G$ ' and viscous $G$ ' moduli (linear regime) is justified. Fig.2b gathers the evolution of $G^{\prime}$ and $G^{\prime}$ ' versus frequency at low strain amplitude versus frequency. The shear modulus $G$ ' dominates the viscous component. Both moduli are weakly dependent on the frequency which is characteristic of a (linear) solid-like response, in agreement with the in-phase wave displayed in Fig.2a.

This result highlights the efficiency of the protocol associating a total wetting to a weak mechanical stress, enabling for the first time, to measure shear elasticity in water at a hundred microns length scale and at such small frequencies.

\subsection{From solid-like to conventional viscous behaviour: strain dependence of the mechanical response.}

The solid-like wave is delicate and can easily shear-melt by increasing the strain amplitude. The evolution of the mechanical response on increasing the strain amplitude is presented on Fig.3a. At low strain amplitude, both input and output waves are close in-phase $\left(\left(\Delta \phi<8^{\circ}\right)\right.$ indicating a major elastic component $\left(G^{\prime}\right)$. The elastic regime corresponds to the "red" zone. In this regime, the elastic modulus $G^{\prime}(\omega)$ is higher than the viscous modulus $G^{\prime \prime}(\omega)$ (the viscous component $(G$ ”) contributes to about $1 \%$ of the total modulus $\left.G=\sqrt{ }\left(G^{, 2}+G^{, 2}\right)\right)$.

At higher strain amplitudes, both elastic and viscous moduli collapse with a more pronounced fall for the elastic modulus and leading to a crossover from a solid-like to a viscous-like behaviour. At high strain amplitude, the viscous modulus dominates (blue zone) and the elastic component becomes negligible with respect to the total modulus (less than $1 \%$ of $G$ at $30 \mathrm{rad} / \mathrm{s}$ ).

As a result, the usual viscous behaviour of water is recovered on wetting substrates, by applying high strain amplitudes, thus away from mechanical equilibrium conditions. It shows that a linear solid-like regime exists prior to the conventional flow regime. A similar strain dependence scheme has been reported for other liquids and melts and by different authors[1-6] confirming that the shear elasticity in the liquid state can be easily lost in favour of the usual flow behaviour.

Fig.3b details the mechanism of transition from solid-like to viscous behaviour. At low shear strain, both input (shear strain) and output (shear stress) waves are quasi-superimposable (Fig.3b left). At intermediate strain rates $\left(\gamma_{0}=10 \%\right.$, middle figure of Fig.3c), the output wave is no more sinusoidal but distorted with apparent harmonics. The occurrence of the distorted signal coincides with the entrance in the non-linear regime where the values of $G^{\prime}$ and $G$ "' are strongly dependent on the strain rate. Since the signal is no more sinusoidal, a modeling in simple terms of $G$ ' and $G$ ', is not strictly meaningful. The wave distortion shows harmonics that can be interpreted as the result from a truncation/recombination of the primitive wave. This effect has to be typically related to a release process of the elastic energy stored in the sample.

At large shear strain $\left(\gamma_{0}>5000 \%\right)$, the wave recovers a quasi-sinusoidal shape with a phase advance of about $\Delta \phi=\pi / 2$ (Fig.3b at right). This output sin wave can be modeled in terms $G$ ”. The associated spectrum displays a viscous behaviour as indicated in the dynamic spectrum measured at $\gamma_{0}=5000 \%$. 
The curve obeys to the $\omega$-scaling with a slope $\eta=10^{-3} \mathrm{~Pa}$.s, of the order of the tabulated dynamic viscosity value[16]. It is interesting to note that the evolution from the low strain amplitude sinusoidal wave to the large strain amplitude sinusoidal-like wave does not correspond to a simple shift of the phase but to a strong modification of the signal.

\subsection{Stress relaxation measurements:}

Another and independent procedure to evidence the solid-like character contained in the liquid water is the relaxation measurement. The sample placed between two surfaces is submitted to a constant shear strain $\gamma_{0}$ by moving one surface at the time $t=0$. The sample transmits the shear stress by contact to the second surface that is coupled to a sensor. This shear stress $\sigma(t)$ is measured versus time. Fig.4a shows the corresponding stress relaxation modulus $G(t)=\sigma(t) / \gamma_{0}$ measured on wetting boundary conditions, at room temperature and $0.125 \mathrm{~mm}$ gap thickness. As can be seen, the liquid does not flow under strain but displays a weak but non-negligible elasticity. The shear stress does not collapse even after more than $10^{3} \mathrm{~s}$. This is a typical solid-like response, coherent with previous observations[1-5]. Increasing the strain amplitude first induces a rapid modulus drop followed by a stress plateau indicating the subsisting resistance of the liquid (Fig.4b). At $\gamma_{0}=10000 \%$, the liquid no more resists and the stress relaxation falls rapidly indicating that the liquid flows. The inset displays the average shear stress as a function of the strain amplitude. The shear stress can be modelled by a continuous decreasing function of the shear strain: $G(\gamma)=G_{0} /\left(1+\left(\gamma / \gamma_{0}\right)^{b}\right)$ with $b=1.05 \pm 0.03$ and $\gamma_{0}=0.9 \pm 0.1$. The extrapolation at zero-strain limit gives the intrinsic stress relaxation modulus (of that sample) $G_{0} \approx 17 \pm 3 \mathrm{~Pa}$. $G_{0}$ is weak but finite, not negligible and of the same order as the low frequency dynamic modulus.

\section{Conclusions:}

Finite low frequency shear elasticity is measured by applying a weak mechanical shear stress at room temperature to liquid water at a sub-millimetre thickness. The shear elasticity is non-ambiguously identified with three different stress measurements presented in Fig.2, 3 and 4 respectively. All of them have been carried out by optimising the motion transfer from the stress source to the sample and from the sample to the sensor. The study of the stress wave shows that a quasi-in phase response to the signal at its source is displayed by water under weak solicitation in the low frequency range. The conservation of the input strain sinusoidal shape to the output shear stress wave proves the solid-like character of the response and enables an analysis in terms of (in-phase: $G^{\prime}$ ) elastic and (out-of-phase: $G$ ") viscous moduli. The examination of the low frequency range $\left(10^{-1}-10^{2} \mathrm{rad} / \mathrm{s}\right)$ indicates in agreement with the shape of the quasi-in-phase signal, that the shear modulus $G^{\prime}$ dominates the mechanical behaviour, with both $G^{\prime}$ and $G$ " being weakly dependent on the frequency. The dominance of the shear modulus indicates a major solid-like response.

The solid-like regime ("Solid" red zone of Fig.3a) is prior in strain to the usual viscous regime ("Liquid" blue zone). Since the lowest strain amplitudes ensure the weakest perturbations of the sample, the elastic response of liquid water is thus the first response of the material. The usual conventional viscous behaviour (carried out without special care of wetting - using generally Aluminium substrates), corresponds to a second linear domain and results from a partial transmission of the stress (weak liquid/surface interactions)[2]. On wetting substrates, the viscous response is recovered by applying very high strain amplitudes (right inset of Fig.3), once the solid-like response has collapsed (as illustrated in figures 3 and 4, and in references[2-5]).

Other measurements (not presented here) carried out at different water thicknesses indicate that the elastic response persists at least from $0.055 \mathrm{~mm}$ up to $0.500 \mathrm{~mm}$, decreasing by increasing the gap and no more measurable at larger thicknesses. We thus probe a macroscopic property which strength depends on the length scale. The dimensional character is also found in molten cements or in foam rheology[17,18]. At $0.125 \mathrm{~mm}$ gap thickness, the low frequency (Hz frequency range) shear modulus is about $20 \mathrm{~Pa}$ (Fig.2b, Fig.3a). This value is extremely weak compared to « conventional » 
solids characterised by elastic moduli of the order of several GPa. It is also weak in comparison to the moduli measured using the atomic vibration of a piezomembrane by Derjaguin: $30 \mathrm{kPa}$ at a frequency of $73 \mathrm{kHz}$ [15] or later by Bund et al: $10 \mathrm{kPa}$ at moderate frequencies $(10 \mathrm{MHz})$ [19]. Finally, compared to the GPa moduli measured by ultra-sonic methods or Brillouin scattering in liquids at $\mathrm{GHz}$ frequencies [20], the low frequency shear elasticity of water appears also insignificant. These elastic properties are however of outmost importance since they tell that water resists to shear flow.

The water elasticity is in agreement with shear moduli measured with this method on other liquids (heptadecane, glycerol, o-terphenyl, polymer melts)[1-5]. It is also coherent with the gel-like behaviour reported in an unentangled melt using a piezomembrane in the same frequency range[6], or with X-ray photon correlation results pointing out the need to add an elastic term for modeling capillary waves in supercooled polypropylene glycol [20]. Nearly static shear elasticity in the liquid state tells that the intermolecular interactions are actually long range. This claim excludes any interpretation of the macroscopic properties based on individual molecular dynamics. Its measurement on water at room temperature confirms that it also at variance with the hypothesis of long lived clusters away from the glass transition[6-7] or of ill-defined supercooled states[21]. In agreement with the pioneering works of Derjaguin[15], the low frequency shear elasticity is an intrinsic condensed matter property and its origin has to be found in the strength and the range of the intermolecular interactions, particularly reinforced by the high H-bonds interconnectivity in case of water. The high degree of intermolecular connectivity at the origin of these long range elastic correlations contributes significantly to the energy of the liquid condensed matter state. Few theoretical approaches consider the condensed matter character of liquids [22,23]. Even fewer approaches consider the occurrence of multi-scale properties. A phenomenological model introducing power laws in reciprocal space [24a] predicts that the intrinsic shear elasticity of a finite volume of liquid is non zero. It also predicts [24b] that the shear modulus measured in a rheology experiment at sufficiently low frequencies is sample size dependent, decreasing with increasing sample size, a behaviour observed by different authors at the sub-millimetre length scale [1-6,15]. Quantitative analysis of some of our present data and data at higher frequencies found in the literature in terms of this model is currently underway.

These experimental results have to be extended and the method certainly improved, but they are already rich of perspectives for a better understanding of the multi scale liquid properties, gelation processes, transport and flow mechanism and points out the necessity to provide bridges between disciplines (micromechanics, microtribology, rheology, microrheology, poroelasticity, surface science). 


\section{References}

[1] Mendil H, Baroni P, Noirez L 2006, Solid-like rheological response of non-entangled polymers in the molten state, Eur. Phys. J. E 1977.

[2] Noirez L, Mendil-Jakani H, Baroni P 2009, The missing parameter in rheology: hidden solid-like correlations in viscous liquids, polymer melts and glass formers, Polymer Int. 58962.

[3] Noirez L, Baroni P, 2010, Revealing the solid-like nature of glycerol at ambient temperature, J. of Molecular Structure, 97216.

[4] Noirez L, Mendil-Jakani H, Baroni P, 2011, Identification of finite shear-elasticity in the liquid state of molecular and polymeric glass-formers, Philosophical Magazine 911977.

[5] L. Noirez, P. Baroni, H. Cao 2012, J. of Mol. Liq. in press.

[6] Collin D, Martinoty P 2002, Dynamic macroscopic heterogeneities in a flexible linear polymer melt, Physica A,320 235.

[7] Steffens W, Patkowski A, Meier G, Fischer E W 1992, Distribution of reorientational times of optically anisotropic molecular liquids from depolarized light scattering studies, J. Chem. Phys. 96 4171; Fischer E W 1993, Light scattering and dielectric studies on glass forming liquids, Physica A 201 183.

[8] Water, A Comprehensive Treatise, Plenum Press, NY and London, F. Franks Editor, 1979.

[9] Hu H-W, Carson G A, Granick S 1991, Relaxation time of confined liquids under shear, Phys.Rev.Lett., 66 2758; Zhu Y, Granick S 2004, Superlubricity: A Paradox about Confined Fluids Resolved, Phys. Rev. Lett., 93 0961011, B. Bhushan, 2005, Nanotribology and nanomechanics, Wear, 2591507.

[10] Israelachvili J N, Mcguiggan P M, Homola A M 1988, Dynamic properties of molecularly thin liquid films, Science 240 189; Israelachvili J N, Pashley R 1982, The hydrophobic interaction is long range, decaying exponentially with distance, Nature $\mathbf{3 0 0} 341$.

[11] Bellissent-Funel M C 2005, Hydrophilic-hydrophobic interplay: from model systems to living systems, Comptes Rendus Géoscience, 337 173; Gabel F, Bellissent-Funel M C 2007, C-Phycocyanin Hydration Water Dynamics in the Presence of Trehalose: An Incoherent Elastic Neutron Scattering Study at Different Energy Resolution, Biophys. J. 92 4054; Protein Dynamics and Hydration Water, in Water: the Forgotten Biological Molecule. D. Le Bihan, Hidenao Fukuyama (2011); L. Bosio L, J. Teixeira J, Bellissent-Funel M C, 1989, Enhanced density fluctuations in water analyzed by neutron scattering, Phys. Rev. A 396612.

[12] Maali A, Cohen-Bouhacina T, Couturier G, Aimé J P 2006, Oscillatory Dissipation of a Simple Confined Liquid, Phys. Rev. Lett., 9686105.

[13] Baroni P, Mendil H, Noirez L 2005, Fr. Pat., 0510988.

[14] Blake T D 1990, Slip between a liquid and a solid: D.M. Tolstoi's (1952) theory reconsidered, Colloids and Surfaces 47135.

[15] Badmaev B B, Bazaron U B, Derjaguin B V, Budaev O R 1983, Measurement of the shear elasticity of polymethylsiloxane liquids, Physica B 122 241, Derjaguin B V, Bazaron U B, Zandanova K T, Budaev O R 1989, The complex shear modulus of polymeric and small-molecule liquids, Polymer, 30 97, Derjaguin B.V., U.V. Bazaron, Lamazhapova, Tsidypov B D 1990, Shear elasticity of lowviscosity liquids at low frequencies, Phys. Rev. A 422255.

[16] Chaplin M, Water Structure and Science, data basis: http://www.lsbu.ac.uk/water/

[17] Bazant Z.P., Scaling of Structural Strengths, Hermes Penton Science, london (2002).

[18] J.P. Heller, M. S. Kuntamukkula 1987, Critical Review oft he Foam Rheology Literature, Ind. Eng. Chem. Res., 26318.

[19] Bund A, Schwitzgebel G 1998, Viscoelastic Properties of Low-Viscosity Liquids Studied with Thickness-Shear Mode Resonators, Anal. Chem. 70 2584; Meister R, Marhoeffer C J, Sciamanda R, Cotter L, Litovitz T 1960, Ultrasonic Viscoelastic Properties of Associated Liquids, J. of Appl. Physics 31 854, Slie W M, Donfor A R, Litovitz T 1966, Ultrasonic Shear and Longitudinal Measurements in Aqueous Glycerol, J. of Chem. Phys. 44 3712, Scarponi F, Gomez L, Fioretto D, 
Palmeri L 2004, Brillouin light scattering from transverse and longitudinal acoustic waves in glycerol, Phys. Rev. B $\mathbf{7 0} 054203$.

[20] Chushkin Y, Caronna C, Madsen A 2008, Low-frequency elastic behavior of a supercooled liquid, EPL 8336001.

[21] Zondervan Z., Xia T., van der Meer H., Storm C., Kulzer F., van Saarlos W., Orrit M. 2008, Soft glassy rheology of supercooled molecular liquids, PNAS 105 4993, Yuan H-F, Plazant M., Demé B., Orrit M. 2012, Crystalline nucleation in supercooled glycerol near the glass transition, J. of Chem. Phys. 13641102.

[22] Granato A V 2009, Mechanical properties of simple condensed matter, Materials Science and Engineering A $\mathbf{5 2 1} 6$.

[23] Dyre J C 1999, Solidity of viscous liquids, Phys. Rev. E 59 2458, Dyre J C 2006, Solidity of viscous liquids. IV. Dyre J C 2006, Density fluctuations, Phys. Rev. E 7421502.

[24a] Volino $\mathrm{F}$ et al 1997, Théorie visco-élastique non-extensive, Annales de Physique, 22 n 1 \& 2 Chapters III-VI, [24b] Volino F, Private communication, manuscript in preparation. 
Figure captions:

Fig.1: Schemes of the sin strain input wave ( - ) and shear stress output response ( - ) (the dotted line represents the $\pi / 4$ phase shift output wave $(---))$, and corresponding low frequency dynamic spectra (logarithmic representation). a) Solid-like behaviour: input and output waves are close in phase: $G^{\prime}>$ $G$ " with $G$ ' and $G$ " weakly dependent on the frequency. b) Viscous behaviour: input and output waves are out of phase with $\Delta \phi>\pi / 4$ (here $\Delta \phi=\pi / 2$ ) with $G^{\prime}$ negligible and G" vanishing with the frequency.

Fig.2: a) Signals corresponding to the input sin strain wave (data points: $=$ ) and output shear stress (data point: $\bullet$ ) wave measured on liquid water at low strain $\left(\gamma_{0}=3 \%\right)$ and at $\omega=1 \mathrm{rad} / \mathrm{s}$. The sin modeling of the input (-) and the output waves (---) are displayed on sides (phase shift $\Delta \phi=9 \pm 1^{\circ}$ ). b) Low strain frequency viscoelastic moduli of liquid water measured at room temperature $\left(21.5^{\circ} \mathrm{C}\right)$, at $0.125 \mathrm{~mm}$ gap thickness and $\gamma_{0}=3 \%$. The shear modulus $G^{\prime}(\bullet)$ is independent of the frequency and higher than $G$ "'(口).

Fig.3: a) Strain dependence of the viscoelastic moduli of liquid water at $\omega=1 \mathrm{rad} / \mathrm{s}$ and at $0.125 \mathrm{~mm}$ gap thickness at room temperature ( $\gamma_{0}$ evolves from $2.5 \%$ up to $7500 \%$ ). The lines are eye-guides. $b$ ) Details of the frequency dependence at weak and at large strain amplitudes $\left(\gamma_{0}=3 \%\right.$ and at $\gamma_{0}=7500 \%$ respectively) and corresponding input sin wave $(\square)$ and output shear stress $(\bullet)$ waves. The frequency dependence at intermediate strain amplitude (500\%) is not displayed since the wave is no more a sin wave. The red line is a sinusoidal fit of the data points. The blue straight line shows the $\omega$-scale of $G$ " indicating a dynamic viscosity $\eta=10^{-3} \mathrm{~Pa}$.s, in agreement with conventional experiments[16]

Fig.4: Time-dependence of the relaxation of the shear modulus $G(t)$ of liquid water at room temperature $\left(\mathrm{T}=21.5^{\circ} \mathrm{C}\right)$ and $0.125 \mathrm{~mm}$ gap thickness: a) Detail of $G(t)$ at $\gamma_{0}=30 \%$. b) Evolution from low $\left(\gamma_{0}=1.75 \%\right)$ up to high strain amplitude $\left(\gamma_{0}=10000 \%\right)$. The inset displays the average value of the stress relaxation modulus versus strain amplitude. The dotted line illustrates the curve fitted by: $G(\gamma)=G_{0} /\left(1+\left(\gamma / \gamma_{0}\right)^{b}\right)$. 


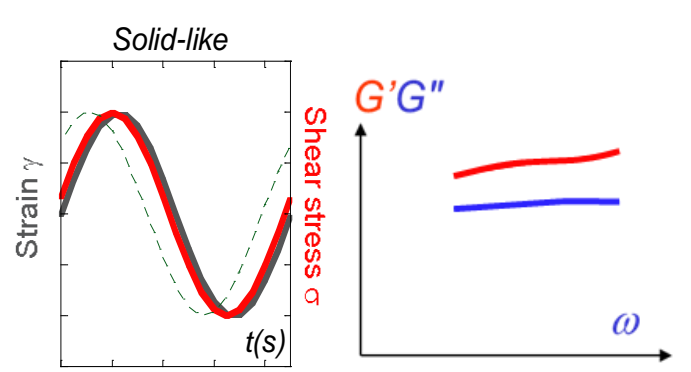

Fig.1a

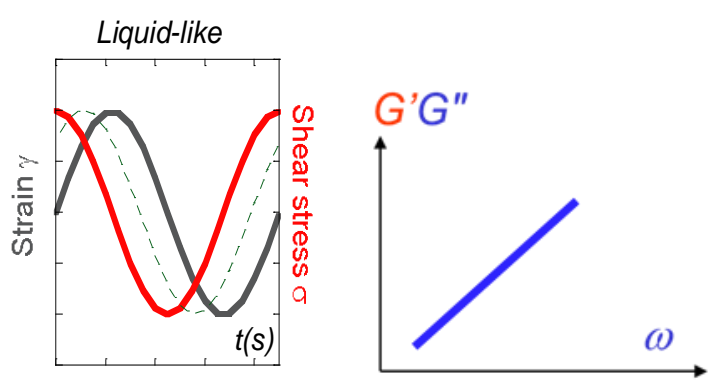

Fig.1b

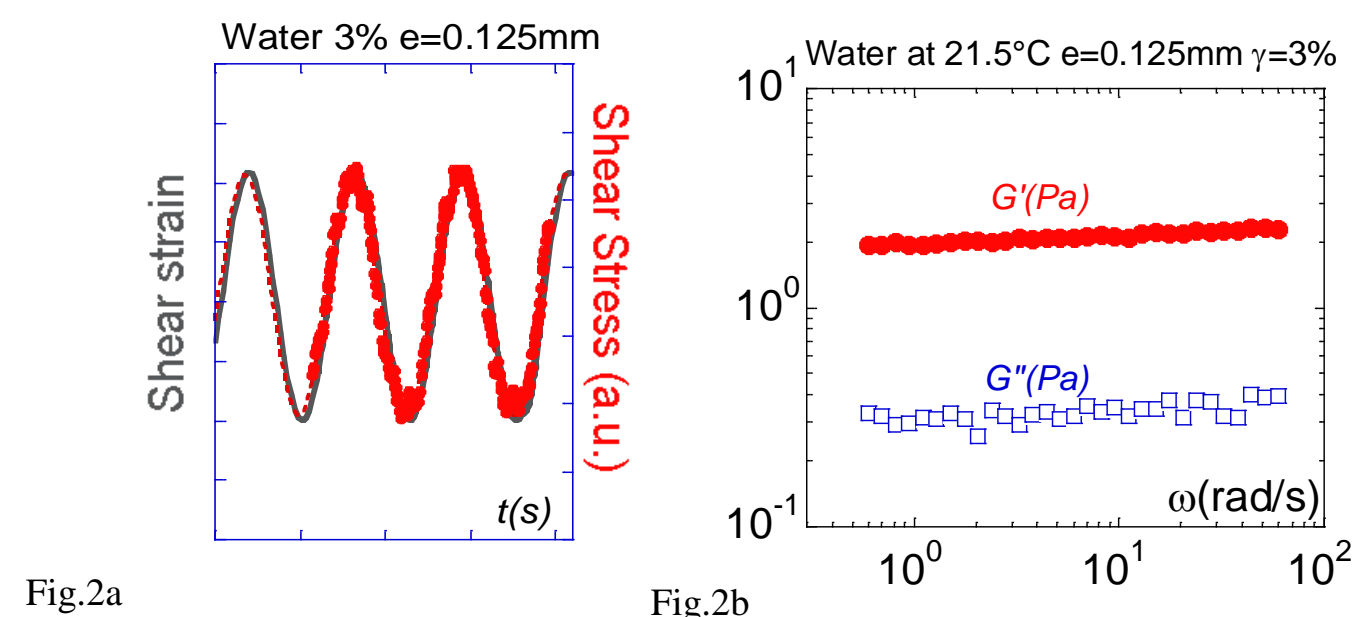

Fig.2a

Fig.2b

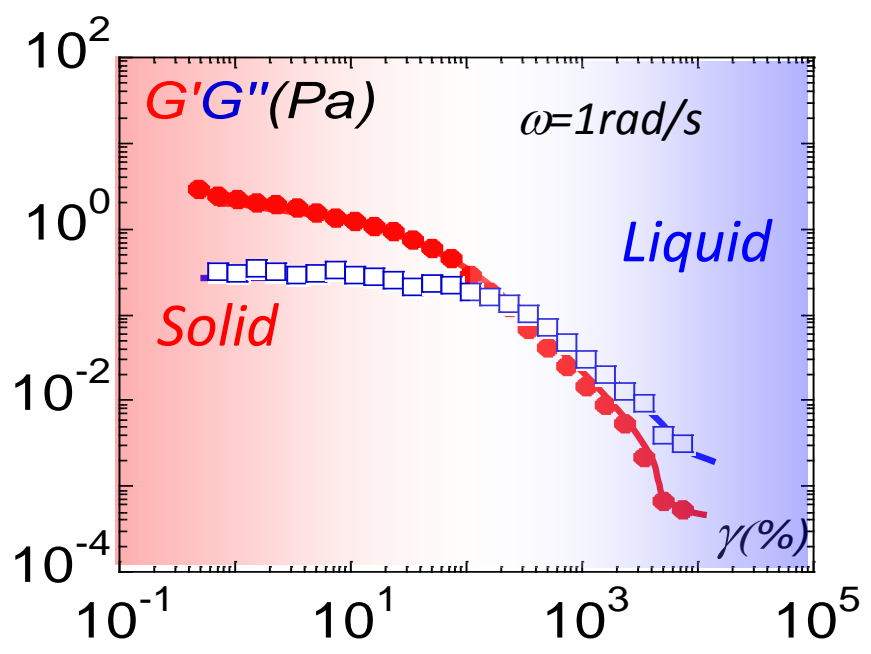

Fig.3a 


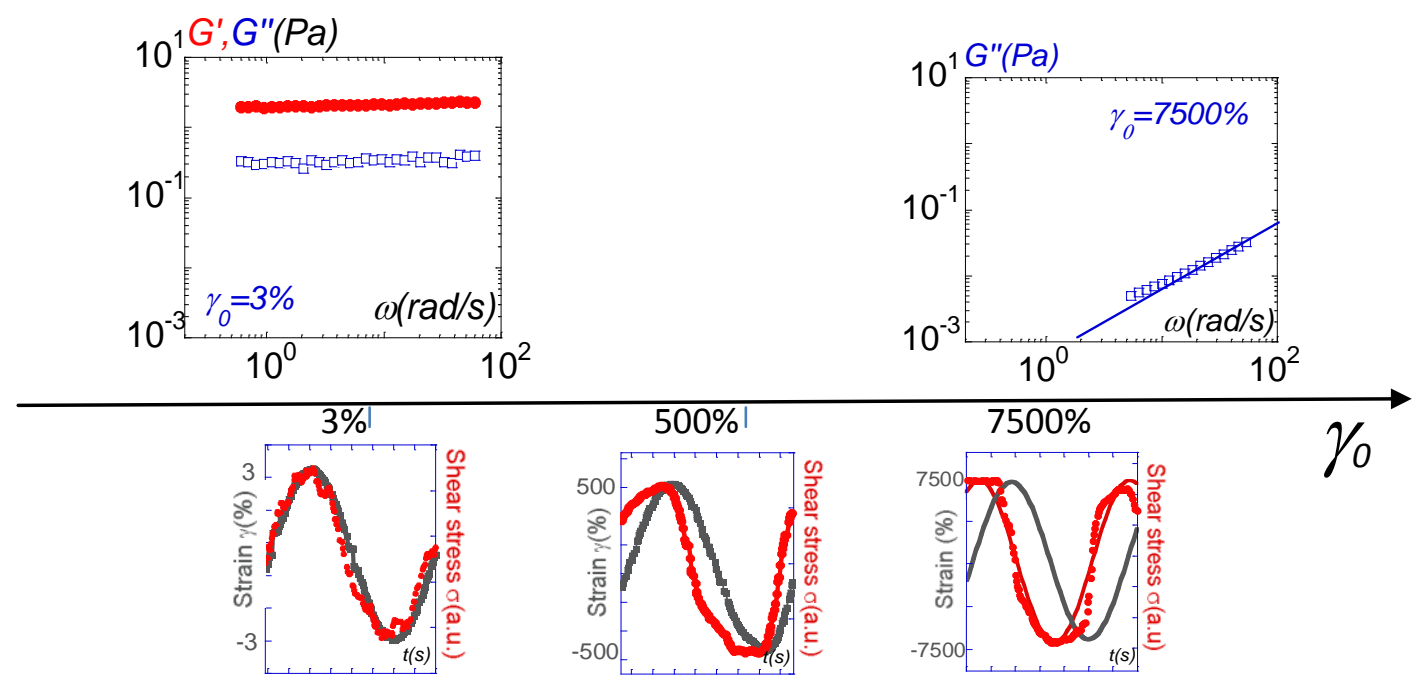

Fig. $3 b$

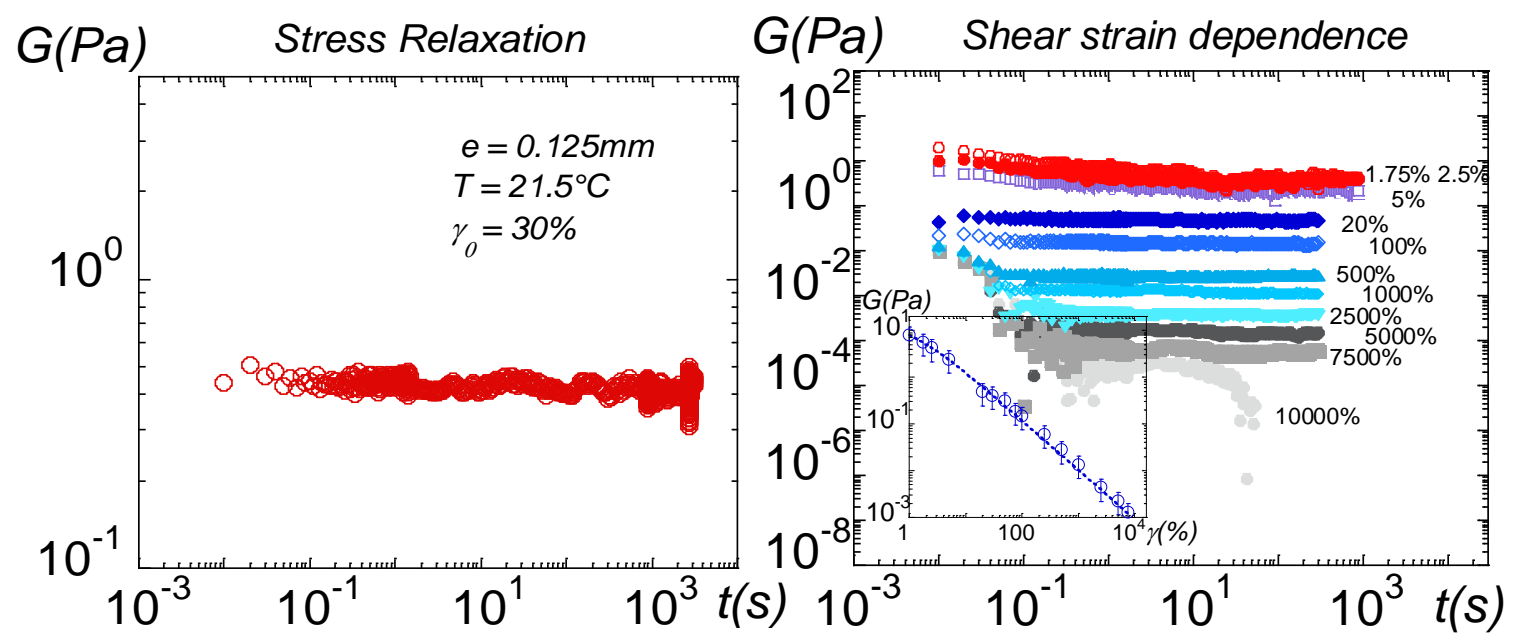

Fig.4a

Fig.4b 